A. Katzer

A. Ince

M. Hahn

M.M. Morlock

W. Steens

\section{Cement mantle defects in total hip arthroplasty: influence of stem size and cementing technique}

Received: 13 May 2007

Accepted: 24 September 2007

Published online: 21 December 2007

\section{A. Katzer (西)}

Orthoclinic Hamburg

Oldesloer Strasse 9, D-22457 Hamburg,

Germany

E-mail: AlexanderKatzer@aol.com

\section{A. Ince}

Trauma Center

General Hospital Hamburg Altona

Hamburg, Germany

\section{Hahn}

Center for Biomechanics

University Medical Center

Hamburg, Germany

\section{M.M. Morlock}

Section for Biomechanics

Hamburg University of Technology

Hamburg, Germany

\section{W. Steens}

Department of Orthopedics

St. Franziskus Hospital

Münster, Germany

\begin{abstract}
The cause of isolated osteolysis in the femoral shaft around stem implants in patients with cemented THR has so far not been established. A number of factors have been considered such as torsional stability of the femoral stem implant, the time of reduction intraoperatively after cementing and iatrogenic and loadinduced defects in the cement mantle. The aim of this in vitro investigation was to determine if the cementing technique or the thickness of the prosthesis stem, and thus its bending strength, influences the formation, extent and localisation of cement mantle defects. In vitro biomechanical loading tests were performed on twelve anatomically shaped femoral stem prostheses of two different thicknesses which were implanted in artificial bone. Six of the implants were fixed by conventional cementing technique, the other six by means of the vacuum technique. Compared with thicker implant stems, the slimmer stems fixed with the conventional cementing technique had a higher number of cracks in the cement mantle. Pore formation was localised predominantly in the interface area between the bone cement and the "cancellous" bone or "cortex" of the artificial bone. This was observed

had been mixed by the conventional method. The thickness of the stems, whether fixed with vacuum-mixed or non-vacuum mixed cement, had no significant influence on the percentage of pore area in the cement. In the nonvacuum mixed cement, there was no significant difference between the percentages of pore area in the proximal and distal parts of the shafts, whereas in the vacuum mixed cement the percentage of pore area was significantly larger in the distal than in the proximal part of the shafts. In the specimens of both stem sizes, the percentage of pore area in the vacuum mixed cement was significantly smaller than in the nonvacuum mixed cement. This explains the greater fatigue strength of vacuum mixed cement. The cyclic loading on the thicker stem prostheses, especially in those fixed with vacuum mixed cement, resulted in fracture between the prosthesis tip and the clamping device due to the local stiffness of the artificial bone. Due to this unfavourable biomechanical property of the artificial bone, further studies will be carried out on human femurs. Nevertheless, in view of the results presented here, the vacuum mixing technique has to recommended as the "state of the art" method in cemented total hip arthroplasty.
\end{abstract} especially in the non-vacuum mixed cement, regardless of stem thickness. Large pores were found mainly in the cement around the thicker stems which
Key words Femoral shaft osteolysis • Cement mantle defect $\cdot$ Cementing technique 


\section{Introduction}

Isolated, aseptic osteolysis in the femoral shaft around femoral implants which are still firmly in place is a rare late complication after implantation of cemented total hip replacements. It only occurs in about $0.2 \%$ of primary implantations [1]. The mean age of the affected patients is lower than that of the patients in comparable cohorts without femoral shaft osteolysis, and the percentage of males is higher [1]. There is no evidence for a correlation between isolated femoral shaft osteolysis and the activity level or body mass index (BMI). Two studies have shown that osteolysis in the femoral shaft occurs mainly in zones 2 (21.6\%), $3(18.1 \%), 5(17.6 \%)$ and $6(29.8 \%)$ of Gruen's classification, as seen on anteroposterior radiographs [1, 2].

The cause of this type of osteolysis has so far not been established. Besides age, gender and activity level, a number of other factors have been considered such as torsional stability of the femoral stem implant, the time of reduction intraoperatively after cementing and iatrogenic and load-induced defects in the cement mantle [1]. In a study by McCormack and Prendergast [3], most cracks in the cement mantle $(90 \%)$ originated from the pores in the bone cement. There is also evidence to suggest a connection between the number of cracks and the porosity of the bone cement $[3,4]$.

The aim of this in vitro investigation was to determine if the thickness of the prosthetic stem, and thus its bending strength, influences the formation, extent and localisation of cement mantle defects. To assess the influence of the cementing technique, we used both vacuum mixed and non-vacuum mixed cement and analysed the results for each cementing technique, as well as the pores and the cement mantle defects themselves.

\section{Material and methods}

Six size 2 (large) and six size 5 (extraslim) left-sided anatomically shaped stem prostheses of the type Endo-Modell Mark III (Waldemar Link, Hamburg, Germany) were used. The straight ribs in the proximal part and the grooves at the distal stem end of this implant serve to enlarge the area available for anchorage. The stem prostheses were $150 \mathrm{~mm}$ long, with a femoral neckshaft angle of $135^{\circ}$ and a cone size of $12-14 \mathrm{~mm}$. The chromium-cobalt head had a diameter of $28 \mathrm{~mm}$. Twelve artificial left femurs (3rd Generation, product no. 3303; Sawbones Europe, Malmö, Sweden) company were also used. The cortex of these artificial femurs consists of a compound of glass fibre and epoxy resin (density, $1.7 \mathrm{~g} / \mathrm{cm}^{3}$; compression strength, $120 \mathrm{MPa}$ ). The cancellous bone is reproduced by means of solid polyurethane foam (density, $0.27 \mathrm{~g} / \mathrm{cm}^{3}$; compression strength, $4.8 \mathrm{MPa}$ ). For resection of the femoral neck, each artificial bone was fitted exactly into a specially made saw guide. The femoral canal was prepared using a gouge and then a series of appropriately sized form rasps. After insertion of a plug into the medullary canal (15 $\mathrm{mm}$ diameter), the prosthesis was cemented in position using Palacos R bone cement (Biomet Merck, Darmstadt, Germany). The bone cement was mixed according to the manufacturer's instructions. Three prostheses of each stem size were implanted with vacuum mixed cement and the other three of each size with non-vacuum mixed cement. After the cement had hardened, control radiographs were made to document the position of the implant and the consistency of the cement mantle. All the femurs were then resected $23 \mathrm{~cm}$ distal of the trochanter minor using a ribbon saw.

A chromium-cobalt head was attached to each stem. The femoral shafts were then fixed in a metal cylinder with Technovit 4004 (fast polymerising, cold curing two-component resin based on methyl-methacrylate; Heraeus Kulzer, Wehrheim/Ts, Germany) using a positioning device of Plexiglass in compliance with the International Standard ISO 7206-4. The Technovit was allowed to harden for at least one hour. The device was then fixed on an xy-table and mounted in a materials testing machine (Prüf und Simulationssysteme, Duisburg, Germany). To minimize friction, force was introduced into the prosthesis head through a polyethylene cup (Fig. 1). A sinusoidal force of pressure ranging from 100 to $2100 \mathrm{~N}$ was introduced through the punch of the materials testing machine. Loading was maintained for two million cycles at a frequency of $7 \mathrm{~Hz}$. This loading programme resulted in fracture in eight of the twelve artificial bones about $1 \mathrm{~cm}$ below the prosthesis tip. In these cases, the proximal femur and the prosthesis were again prepared with Technovit and the mechanical test was continued for the remaining number of cycles. After each set of 1000 cycles, the path of the punch and the force of pressure were recorded.

After completion of the mechanical tests, the artificial femurs were taken out of the metal cylinder and the prosthesis head was removed. Using a high-pressure abrasive water jet (pressure, $300 \mathrm{MPa}$; speed of the jet head, $30 \mathrm{~mm} / \mathrm{min}$; abrasive mass current, $4 \mathrm{~g} / \mathrm{s}$; Flow Europe, Bretten, Germany), three distal and three proximal sections, 10-mm thick, were cut out of each of the twelve specimens orthogonal to the prosthesis stem.

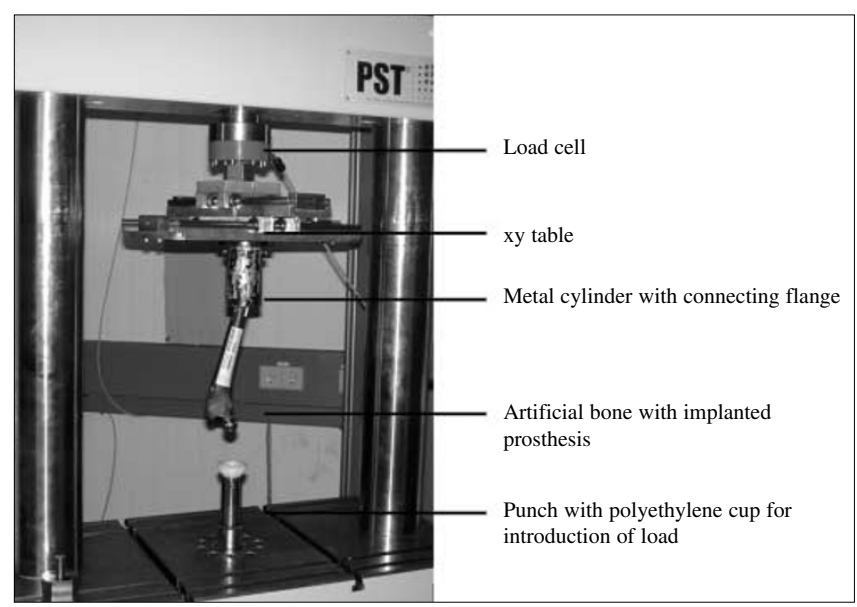

Fig. 1 Test apparatus 


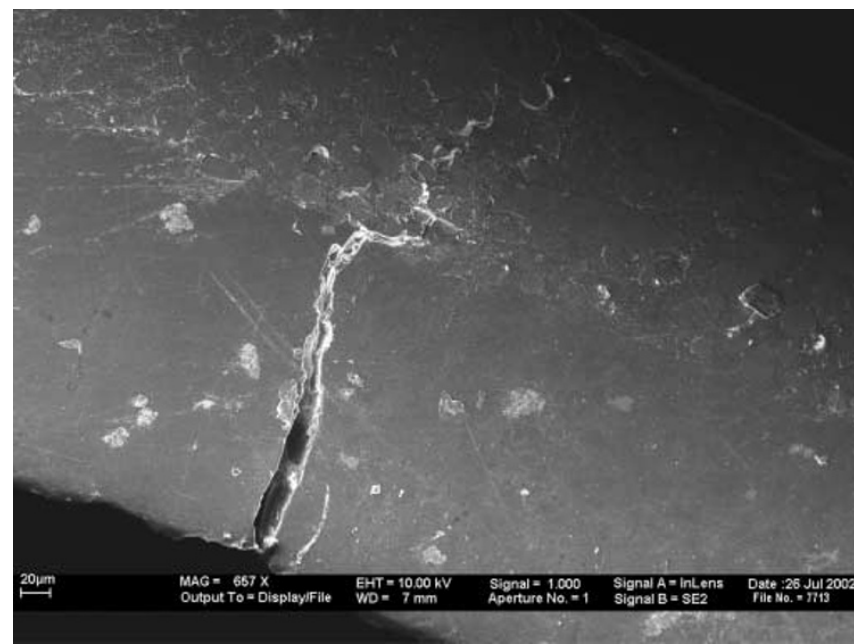

Fig. 2 View under the scanning electron microscope of a sample from section 1 (vacuum mixed cement, stem size 5, 657x magnification). A $150 \mu \mathrm{m}$ crack beginning in the area between the prosthesis and the bone cement is seen

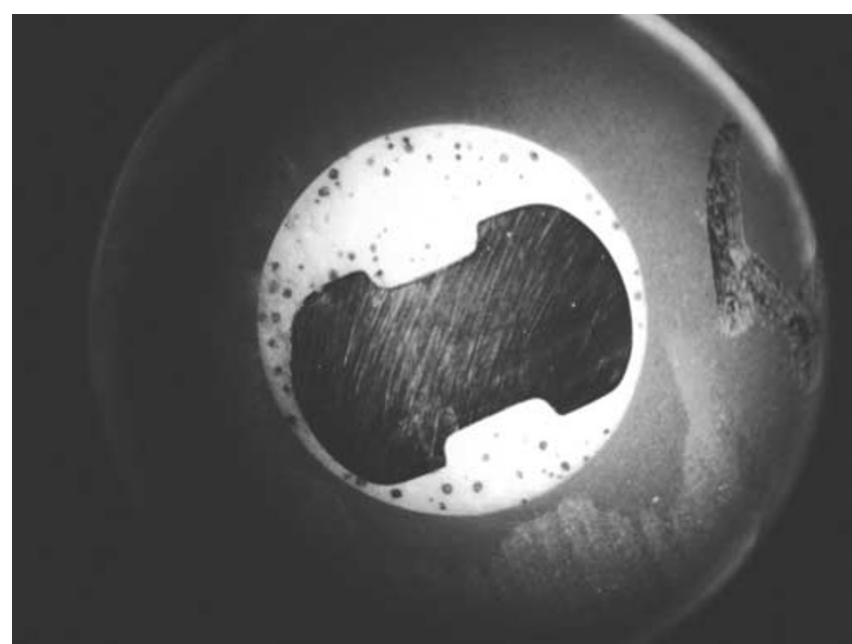

Fig. 3 View of the pores shown with black permanent marker (section 1, proximal area, non-vacuum mixed cement, stem size 2)

As unevenness of the cement surface renders the search for cracks difficult (Fig. 2), both surfaces of each section were polished with $\mathrm{SiC}$ abrasive paper on a polishing machine (Knuth Rotor 2; Struers, Willich, Germany) with simultaneous water rinsing by hand or by means of two automatic sanding machines (EXAKT Apparatebau, Norderstedt, Germany). The grain of the abrasive paper was successively increased from 120 to 4000 so that the final coarseness of the surfaces was about $5 \mu \mathrm{m}$. Both surfaces of each section were then dyed using a black permanent marker and immediately cleaned with ethanol. The good contrast between the pore surface and the rest of the cement surface (Fig. 3) made it possible to determine the pore area automatically using an image analysis system (IBAS 2000 Kontron; CKMedical, Ergolding, Germany). The cement mantle was analysed for section 1 (distal section) and section 6 (proximal section).
The other sections were examined for larger defects only. Two methods were used to determine the percentage of the pore area in relation to the total area of the cement mantle:

1. The sections were placed under a light-optical microscope and photographed with a digital camera. Using an imageprocessing programme (Adobe Photoshop, version 6.0; Adobe Systems, Munich, Germany), the area of cross-section of the pores was marked in black using contrast filters. In a further image, the entire cross-section of the cement mantle was coloured black. The areas of the cross-sections of the pores and of the cement mantle were determined using a different image processing programme (Scion Image, version beta 4.0.2; Scion; Frederick, USA). To calibrate the determination of the area (ratio of the number of pixels to the area), a $1 \mathrm{~mm}^{2}$ black square was photographed in the same way as the sections. The percentage of pore cross-sectional area was then calculated from the ratio of the cross-sectional area of the pores to the cross-sectional area of the cement mantle for both sides of each section.

2. In the second method, the sections were placed under a reflected light microscope (Carl Zeiss, Oberkochen, Germany) connected to an image-analyzing computer. Four representative fields (medial, lateral, anterior and posterior areas of the cement mantle) on each specimen surface with a cross-section of about $10 \mathrm{~mm}^{2}$ were analysed at a magnification of 50x. The percentage of the cross-sectional area of the pores was calculated automatically and was carried out only for the proximal side of each section.

Automatic detection of cracks in the cement mantle was not possible with the image-analyzing computer. This analysis was therefore carried out by two observers independently using a reflected light microscope with a magnification of up to $100 x$. The proximal surfaces of each section were examined systematically for cracks and the position of each crack was entered into a test result record.

The results were evaluated statistically using the $t$ test for independent random samples to prove or disprove the following hypotheses:

1. The size of the prosthesis stem alone, whether fixed by means of vacuum mixed or non-vacuum mixed cement, has no significant influence on the percentage of pore area in relation to the cement mantle area.

2. There is no significant difference in the percentage of pore area in relation to the cement mantle area between the proximal and distal parts of the stem, whether the prosthesis was fixed by means of vacuum mixed or non-vacuum mixed cement.

3. For both stem sizes there is a significant difference between vacuum mixed and non-vacuum mixed cement regarding the percentage of the pore area.

4. For both stem sizes there is no significant difference between vacuum mixed and non-vacuum mixed cement regarding the number of cracks.

5. The size of the prosthesis stem alone has no significant influence on the number of cracks.

Statistical analysis was performed using SPSS (version 9.0.1; SPSS, Chicago, USA). The level of significance was set at $p<0.05$. 


\section{Results}

Under the cyclic loading in the material test machine, eight of the twelve test bones fractured prematurely between the prosthesis tip and the Technovit embedding. In the eight bones that fractured, five of the prostheses had been fixed with vacuum mixed cement and three with non-vacuum mixed cement. All three bones in which a prosthesis size 2 (large) had been implanted and fixed with vacuum mixed cement fractured. This might be due to the high flexural strength of the artificial bone, resulting from the thicker prosthesis and fixation by the vacuum mixing cement technique. The maximum deflection occurred between the end of the cement mantle and the Technovit embedding and finally resulted in fracture.

Pores were localised mainly in the interface between the bone cement and the "cancellous bone" or "cortex" of the artificial bone. This was especially so in the distal sections of both prosthesis sizes which had been fixed with non-vacuum mixed cement. Large pores ( $>2 \mathrm{~mm}$ diameter) were observed mainly in the femurs with a size 2 (large) implant and conventionally mixed cement.

Inclusions with a diameter greater than $2 \mathrm{~mm}$ were excluded from the analysis of the percentage of the pore area. Results from the two methods of analysis are shown in Table 1. The thickness of the stem, whether fixed with vacuum mixed $\left(\mathrm{p}_{1}=0.584, \mathrm{p}_{2}=0.98\right)$ or non-vacuum mixed cement $\left(\mathrm{p}_{1}=0.349, \mathrm{p}_{2}=0.404\right)$, had no significant influence on the percentage of pore area (Table 2). Hypothesis 1 was therefore confirmed. In the specimens with non-vacuum mixed cement, there was no significant difference between the percentages of pore area in the proximal and distal shaft regions $\left(p_{1}=0.583, p_{2}=0.628\right)$. In the specimens with vacuum mixed cement, the percentage of pore

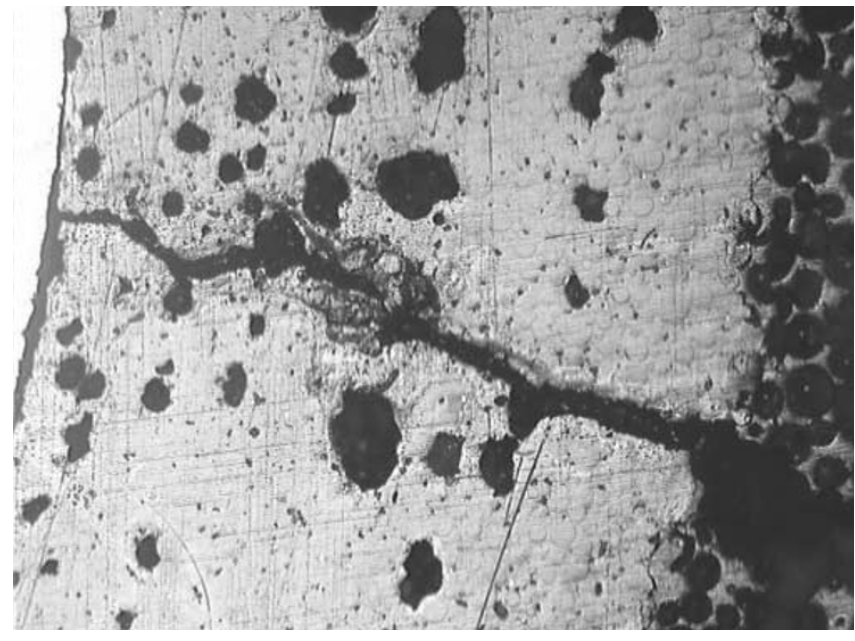

Fig. 4 Crack detection (magnification, 100x)

area in the distal section was significantly larger than in the proximal section $\left(p_{1}=0.010, p_{2}=0.014\right)$. Hypothesis 2 was therefore disproved for vacuum mixed cement. The percentage of pore area in both size $2 \quad\left(p_{1}=0.030\right.$, $\left.p_{2}=0.004\right)$ and size $5\left(p_{1}=0.048, p_{2}=0.001\right)$ prostheses was significantly smaller in the specimens with vacuum mixed cement than in those with non-vacuum mixed cement. Hypothesis 3 was therefore confirmed.

The two independent observers who carried out the analysis of the cracks came to the same results (Fig. 4). A statistical analysis was not performed here as the total number of cracks was very small. Therefore hypotheses 4 and 5 could not be confirmed statistically. Nevertheless, all cracks were found in specimens with non-vacuum mixed cement. Four out of five cracks were found in the bones with the smaller-size prostheses, mainly in the

Table 1 Percentage pore area in relation to stem size, location of the section, cementing technique and method of analysis. Values are mean (SD)

\begin{tabular}{|c|c|c|c|c|c|c|c|c|}
\hline & \multicolumn{4}{|c|}{ Stem size 2} & \multicolumn{4}{|c|}{ Stem size 5} \\
\hline & \multicolumn{2}{|c|}{ Proximal section } & \multicolumn{2}{|c|}{ Distal section } & \multicolumn{2}{|c|}{ Proximal section } & \multicolumn{2}{|c|}{ Distal section } \\
\hline & $\mathrm{V}$ & NV & $\mathrm{V}$ & NV & $\mathrm{V}$ & $\mathrm{NV}$ & $\mathrm{V}$ & NV \\
\hline Method 1 & $2.08(0.8)$ & $6.68(2.68)$ & $5.02(1.77)$ & $7.34(3.21)$ & $2.31(1.25)$ & $8.69(4.62)$ & $3.71(0.61)$ & $12.0(11.19)$ \\
\hline Method 2 & $1.62(0.37)$ & $8.79(1.42)$ & $3.72(1.38)$ & $8.35(5.55)$ & $1.49(0.88)$ & $7.78(1.77)$ & $3.90(1.99)$ & $6.61(0.56)$ \\
\hline
\end{tabular}

$V$, vacuum mixed cement; $N V$, non-vacuum mixed cement

Table 2 Significance levels $(p)$ of influence of stem size on the average percentage of pore area in relation to cementing technique

\begin{tabular}{lrr}
\hline & Stem size 2 & Stem size 5 \\
\hline Vacuum mixed (V) & $p_{1}=0.584$ & $p_{2}=0.98$ \\
Non-vacuum mixed (NV) & $p_{1}=0.349$ & $p_{2}=0.405$ \\
\hline
\end{tabular}


immediate vicinity of the prosthetic ribs, and the cracks were found mainly in the proximal part of the stem.

\section{Discussion}

Preparation of the implant bed for the thicker prostheses turned out to be an arduous undertaking using the standard instrumentarium. The cancellous bone and cortex of the artificial bone were difficult to form due to their brittleness. It would have been an advantage to have had artificial bones of a larger size than is currently commercially available. The larger-sized stem prostheses implanted with vacuum mixed cement caused an extreme increase in the stiffness of the bone around the implant. This led to fracture of the bone between the prosthesis tip and the clamping device which in turn resulted in unequal free clamping lengths. Similar mechanisms may be a cause of periprosthetic femur fractures which occur as a result of only trivial trauma [5].

The porosity of the artificial cancellous bone is lower than that of human cancellous bone. This reduced the penetration of the bone cement into the cancellous bone and the bonding between the bone cement and the cancellous bone therefore did not correspond to the situation in real human bone. Race et al. [6] implanted cemented prostheses in human femurs. They observed formation of cracks mainly in the interface between the cancellous bone and the cement. Many of the cracks were localised in the cement between the trabeculae.

In our study, cement pores were found mainly in the contact area between the bone cement and the cancellous and cortical bone. This is due to the direction of polymerisation of the bone cement from the prosthesis towards the artificial bone under different temperatures in the bone, bone cement and the prosthesis. Inclusions with a diameter greater than $2 \mathrm{~mm}$ were excluded from the analysis of the percentage of the pore area, since these large inclusions resulted from air that was not able to escape during insertion of the cement and implantation of the prosthesis. The thickness of the prosthesis stem alone, whether fixed with vacuum mixed or non-vacuum mixed cement, had no significant influence on the percentage of pore area. Hypothesis 1 was therefore confirmed.

Culleton et al. [7] investigated the cement mantle of a revised prosthesis and found large inclusions which had probably formed during the implantation procedure. In our specimens, large inclusions of air were found in both types of cement mixture, mainly in the distal region. Isolated inclusions were found in the proximal region between the ribs of the thicker prostheses. In the specimens with nonvacuum mixed cement, there was no significant difference between the percentages of pore area in the proximal and distal shaft regions. In the specimens with vacuum mixed cement, the percentage of pore area in the distal section was significantly larger than in the proximal section. Hypothesis 2 was therefore disproved for vacuum mixed cement.

In the specimens of both stem sizes, the percentage of pore area in vacuum mixed cement was significantly smaller than in non-vacuum mixed cement. Hypothesis 3 was therefore confirmed. In comparison with the conventional mixing method, vacuum mixing reduced the pore area by about $70 \%-80 \%$. The investigation [8] of pore areas in Simplex P bone cement [9] mixed by different techniques lead to similar results (vacuum mixed cement, $3.58 \% \pm 6.16 \%$; non-vacuum mixed cement, $4.96 \% \pm 6.21 \%$ ). For Palacos bone cement mixed at a pressure of $72 \mathrm{kPa}$ under atmospheric pressure, a pore area percentage of $2.16 \%$ in comparison with $7.07 \%$ was reported when the cement was mixed by the non-vacuum technique. This explains the greater fatigue strength of vacuum mixed cement.

As weak points in the bone cement, pores promote the formation of cracks, regardless of the size of the implanted prosthetic stem $[10,11]$. Due to the very small cracks, analysis of the specimens revealed for both stem sizes and mixing techniques no significant differences regarding the number of cracks. Therefore hypotheses 4 and 5 could neither be confirmed nor disproved statistically. Nevertheless, all cracks were found in non-vacuum mixed cement. Four out of five cracks were found in the bones with the smaller prostheses, mainly in the immediate vicinity of the prosthetic ribs, and the cracks were found mainly in the proximal part of the stem.

The cause for this localization is certainly, as in vivo, the proximal introduction of load and the greater degree of local weight-bearing on the cement mantle in this region. Race et al. [6] also found that the total length of cracks was significantly greater in the proximal cement mantle. A larger number of cracks was recorded for the slimmer prostheses. This has already been observed in connection with the development of femoral shaft osteolysis in patients with still firmly fixed prosthetic stems [1].

In three cases, the cracks were localised in the immediate vicinity of the longitudinal ribs on the prostheses. This result correlates with that of Jasty et al. [12] who showed in a study of cadaveric femurs that the largest cracks originated from the edges of the prostheses. The question as to whether the cracks developed as a result of mechanical stress or of tension during cement contraction will have to be addressed in further investigations. Lennon and Prendergast came to the conclusion that the tension resulting from cement shrinkage is already sufficient to cause cracks [13]. Furthermore, debonding, during which the prosthetic surface becomes loosened from the bone cement, can also promote formation of cracks and then result in areas of osteolysis [14-16]. 


\section{References}

1. Katzer A, Behnke B, von Foerster G (2001) Osteolysis of the femoral shaft after implantation of a cemented hip prosthesis. J Biomech 34:68

2. Gruen TA, McNeice GM, Amstutz HC (1979) "Modes of failure" of cemented stem-type femoral components: a radiographic analysis of loosening. Clin Orthop 141:17-27

3. McCormack BA, Prendergast PJ (1999) Microdamage accumulation in the cement layer of hip replacements under flexural loading. J Biomech 32:467-475

4. McCormack BA, Prendergast PJ, Gallagher DG (1996) An experimental study of damage accumulation in cemented hip prostheses. Clin Biomech 11:214-219

5. Katzer A, Ince A, Wodtke J, Loehr J.F. (2006) Component exchange in treatment of periprosthetic femoral fractures. J Arthroplasty 21:572-579

6. Race A, Miller MA, Ayers DC, Mann KA (2003) Early cement damage around a femoral stem is concentrated at the cement/bone interface. J Biomech 36:489-496
7. Culleton P, Prendergast PJ, Taylor D (1993) Fatigue failure in the cement mantle of an artificial hip joint. Clin Mater 12:95-102

8. Fish DN, Hoffman HM, Danziger LH (1992) Antibiotic impregnated cement use in US hospitals. Am J Hosp Pharm 49:2469-2474

9. Kühn KD (2000) Bone Cements. Springer-Verlag Berlin, pp 21-26

10. Dunne NJ, Orr JF, Mushipe MT, Eveleigh RJ (2003) The relationship between porosity and fatigue characteristics of bone cements. Biomaterials 24:239-245

11. Maloney WJ, Schmalzried T, Harris WH (2002) Analysis of long-term cemented total hip arthroplasty retrievals. Clin Orthop 405:70-78

12. Jasty M, Maloney WJ, Bragdon CR, O'Connor DO, Haire T, Harris WH (1991) The initiation of failure in cemented femoral components of hip arthroplasties. J Bone Joint Surg $\mathrm{Br}$ 73:551-558
13. Lennon AB, Prendergast PJ (2002) Residual stress due to curing can initiate damage in porous bone cement: experimental and theoretical evidence. J Biomech 35:311-321

14. Berry DJ, Harmsen WS, Ilstrup DM (1998) The natural history of debonding of the femoral component from the cement and its effect on long-term survival of Charnley total hip replacements. J Bone Joint Surg Am 80:715-721

15. Verdonschot N, Huiskes R (1997) Cement debonding process of total hip arthroplasty stems. Clin Orthop 336:297-307

16. Wroblewski BM (1999) The natural history of debonding of the femoral component from the cement and its effect on long-term survival of Charnley total hip replacements. J Bone Joint Surg Am 81:1501 